Pacific Journal of Mathematic 


\title{
MAXIMAL SUBMONOIDS OF THE TRANSLATIONAL HULL
}

\author{
Mario Petrich
}

Maximal submonoids of a semigroup have recently attracted attention in semigroup literature. This is particularly true for the semigroup $\mathscr{B}(X)$ of binary relations on a set. The interesting results of Zareckii in this direction point to the fact that some of these statements pertain to the more general situation of the translational hull of a Rees matrix semigroup. More generally, we consider here maximal submonoids of the translational hull of a regular semigroup.

The first, and the main, theorem in this paper says that if $\omega$ is an idempotent bitranslation of a regular semigroup $S$, then $\omega \Omega(S) \omega \cong$ $\Omega(\omega S \omega)$; here $\omega \Omega(S) \omega$ is a maximal submonoid of $\Omega(S)$. The second theorem pertains to subdirect irreducibility of certain subsemigroups of the translational hull of a Rees matrix semigroup. Finally, the third theorem concerns regular semigroups in which every maximal submonoid is a retract. These results have a number of consequences. The paper ends with several examples of concrete semigroups to which some of the preceding results are applied.

We start with a list of needed definitions and simple results. Let $S$ be a semigroup. A function $\lambda$ (resp. $\rho$ ), written on the left (resp. right) is a left (resp. right) translation of $S$ if $\lambda(x y)=(\lambda x) y$ (resp. $(x y) \rho=x(y \rho)$ ) for all $x, y \in S$. The set $\Lambda(S)$ (resp. $P(S)$ ) of all left (resp. right) translations of $S$ under composition $(\lambda \lambda)^{\prime} x=$ $\lambda\left(\lambda^{\prime} z\right)$ (resp. $\left.x\left(\rho \rho^{\prime}\right)=(x \rho) \rho^{\prime}\right)$ is a semigroup. The pair $(\lambda, \rho) \in \Lambda(S) \times$ $P(S)$ is a bitranslation of $S$ if $x(\lambda y)=(x \rho) y$ for all $x, y \in S$; the subsemigroup of $\Lambda(S) \times P(S)$ consisting of all bitranslations is the translational hull $\Omega(S)$ of $S$. Its elements will be usually written as $\omega=(\lambda, \rho)$, where $\omega$ is considered as a bioperator on $S$. For any $s \in S$, the function $\lambda_{s}$ (resp. $\rho_{s}$ ) defined by $\lambda_{s}=s x$ (resp. $x \rho_{s}=x s$ ) for all $x \in S$, is the inner left (resp. right) translation and $\pi_{s}=$ $\left(\lambda_{s}, \rho_{s}\right)$ is the inner bitranslation of $S$ induced by $s$. The set $\Pi(S)=\left\{\pi_{s} \mid s \in S\right\}$ is an ideal of $\Omega(S)$ called its inner part. The mapping $\pi: s \rightarrow \pi_{s}$ is the canonical homomorphism of $S$ into $\Omega(S)$. It is one-to-one if and only if $S$ is weakly reductive. In such a case for any $(\lambda, \rho),\left(\lambda^{\prime}, \rho^{\prime}\right) \in \Omega(S), s \in S$, we have $(\lambda s) \rho=\lambda(s \rho)$, and thus all parentheses may be omitted.

An element $s \in S$ is regular if $s=s t s$ for some $t \in S$; if also $t=t s t$, then $t$ is an inverse of $s$. A semigroup in which every element is regular is a regular semigroup. Note that every regular 
element has an inverse, and that a regular semigroup is weakly reductive, and hence the canonical homomorphism above is one-toone. A semigroup $S$ is completely regular if every element of $S$ has an inverse with which it commutes (equivalently, $S$ is a union of groups).

An element $e$ of $S$ is idempotent if $e^{2}=e$; the set of all idempotents of $S$ will be denoted by $E_{S}$. If $e \in E_{S}$, then the set $e S e=$ $\{e s e \mid s \in S\}$ is the set of all elements of $S$ having $e$ as a (two-sided) identity, and is thus called a maximal submonoid of $S$ (since a semigroup with an identity element is called a monoid). It is easy to see that every maximal submonoid of a regular semigroup is again a regular semigroup. If $\omega=(\lambda, \rho) \in E_{\Omega(S)}$, the above definitions and conventions yield

$$
\omega S \omega=\{\lambda s \rho \mid s \in S\}=\{s \in S \mid s=\lambda s=s \rho\} .
$$

If $I$ is an ideal of $S$, then $S$ is an (ideal) extension of $I$; $S$ is a dense extension of $I$ if the equality relation on $S$ is the only congruence on $S$ whose restriction to $I$ is the equality relation; if $S$ is a maximal dense extension of $I$, then $I$ is a densely embedded ideal of $S$. For a weakly reductive semigroup $S, \Pi(S)$ is a densely embedded ideal of $\Omega(S)$.

The proofs of the above statements as well as the concepts used in the paper but not defined can be found in the book [5]. This reference as well as the survey article [2] contain a comprehensive collection of results concerning the translational hull.

2. The main theorem. This result gives a suitable isomorphic copy of maximal submonoids of the translational hull of a regular semigroup.

THEOREM 1. Let $S$ be a regular semigroup. If $\omega \in E_{\Omega(S)}$, then the function $\chi$ defined by

$$
\chi:(\varphi, \psi) \longrightarrow\left(\left.\varphi\right|_{\omega, \omega},\left.\psi\right|_{\omega, \omega}\right) \quad((\varphi, \psi) \in \omega \Omega(S) \omega),
$$

is an isomorphism of $\omega \Omega(S) \omega$ onto $\Omega(\omega S \omega)$.

Proof. Let $\omega=(\lambda, \rho)$ and note that

$$
\omega \Omega(S) \omega=\{(\varphi, \psi) \in \Omega(S) \mid \varphi=\lambda \phi=\varphi \lambda, \lambda=\rho \psi=\psi \rho\} .
$$

Next let $(\varphi, \psi) \in \omega \Omega(S) \omega$. For any $x \in \omega S \omega$, using (1) and (2) we have

$$
\varphi x=(\lambda \varphi)(x \rho)=\lambda(\varphi x) \rho \in \omega S \omega
$$


so that $\left.\varphi\right|_{\omega S \omega}$ maps $\omega S \omega$ into itself. Similarly $\left.\psi\right|_{\omega S \omega}$ has the same property. It then follows without difficulty that $\chi$ is a homomorphism of $\omega \Omega(S) \omega$ into $\Omega(\omega S \omega)$.

Next let $(\varphi, \psi),\left(\phi^{\prime}, \psi^{\prime}\right) \in \omega \Omega(S) \omega$ and assume that $(\varphi, \psi) \chi=$ $\left(\varphi^{\prime}, \psi^{\prime}\right) \chi$. Let $x \in S$; there exists $u \in S$ such that $\lambda x=(\lambda x) u(\lambda x)$. Then $\lambda(x u) \rho \in \omega S \omega$ and

$$
\begin{aligned}
\varphi x & =(\varphi \lambda) x=\varphi(\lambda x)=\varphi[(\lambda x) u(\lambda x)]=[\varphi(\lambda(x u) \rho)] x \\
& =\left[\varphi^{\prime}(\lambda(x u) \rho)\right] x=\varphi^{\prime}[(\lambda x) u(\lambda x)]=\varphi^{\prime}(\lambda x)=\left(\varphi^{\prime} \lambda\right) x=\varphi^{\prime} x
\end{aligned}
$$

so that $\varphi=\varphi^{\prime}$; analogously $\psi=\psi^{\prime}$. Consequently $\chi$ is one-to-one.

Next let $(\varphi, \psi) \in \Omega(\omega S \omega)$. Define $\phi^{\prime}$ and $\psi^{\prime}$ on $S$ by

$$
\begin{array}{lll}
\varphi^{\prime} x=[\varphi(\lambda(x u) \rho)] x & \text { if } & \lambda x=(\lambda x) u(\lambda x), \\
x \psi^{\prime}=x[(\lambda(v x) \rho) \psi] & \text { if } & x \rho=(x \rho) v(x \rho) .
\end{array}
$$

We will show first that the definition of $\phi^{\prime}$ is independent of the choice of the element $u$. Hence assume that

$$
\lambda x=(\lambda x) u(\lambda x)=(\lambda x) t(\lambda x) .
$$

Then

$$
\lambda(x u) \rho=(\lambda x)(u \rho)=(\lambda x) t(\lambda x)(u \rho)=[\lambda(x t) \rho][\lambda(x u) \rho]
$$

so that

$$
\begin{aligned}
{[\varphi(\lambda(x u) \rho)] x } & =\{\varphi[((\lambda(x t) \rho)(\lambda(x u) \rho)]\} x=[\varphi(\lambda(x t) \rho)][\lambda(x u) \rho] x \\
& =[\varphi(\lambda(x t) \rho)](\lambda x) u(\lambda x)=[\varphi(\lambda(x t) \rho)](\lambda x)
\end{aligned}
$$

which evidently implies independence of $\varphi^{\prime}$ on the choice of $u$. Similarly the definition of $\psi^{\prime}$ is independent of the choice of $v$.

Now let $x, y \in S, \lambda x=(\lambda x) u(\lambda x), \lambda(x y)=\lambda(x y) w \lambda(x y)$. Using (3), we obtain

$$
\begin{aligned}
\left(\varphi^{\prime} x\right) y & =[\varphi(\lambda(x u) \rho)] x y=[\varphi(\lambda(x u) \rho)](\lambda x) y \\
& =[\varphi(\lambda(x u) \rho)] \lambda(x y) w \lambda(x y)=[\varphi(\lambda(x u) \rho)][\lambda(x y w) \rho] x y \\
& =\{\varphi[(\lambda(x u) \rho)(\lambda(x y w) \rho)]\} x y \\
& =\{\varphi[(\lambda(x u) \rho)(\lambda x)(y w) \rho)]\} x y \\
& =\{\varphi[(\lambda x) u(\lambda x)(y w) \rho]\} x y \\
& =\{\varphi[(\lambda x)(y w) \rho]\} x y=\left[\varphi(\lambda(x y w) \rho] x y=\phi^{\prime}(x y) .\right.
\end{aligned}
$$

Hence $\varphi^{\prime}$ is a left translation of $S$, a symmetric proof shows that $\psi^{\prime}$ is a right translation of $S$.

Let $x, y \in S, x \rho=(x \rho) s(x \rho), \lambda y=(\lambda y) z(\lambda y)$. Then 


$$
\begin{aligned}
x\left(\varphi^{\prime} y\right) & =x[\varphi(\lambda(y z)) \rho)] y=x[(\lambda \varphi)(\lambda(y z) \rho)] y \\
& =x\{\lambda[(\varphi(y z) \rho)]\} y=(x \rho)[\varphi(\lambda(y z) \rho)] y \\
& =(x \rho) s(x \rho)[\varphi(\lambda(y z) \rho)] y=x[\lambda(s x) \rho][\varphi(\lambda(y z) \rho)] y \\
& =x[(\lambda(s x) \rho) \psi][\lambda(y z) \rho] y=x[(\lambda(s x) \rho) \psi](\lambda y) z(\lambda y) \\
& =x[(\lambda(s x) \rho) \psi](\lambda y)=x\{[(\lambda(s x) \rho) \psi] \rho\} y \\
& =x[(\lambda(s x) \rho)(\psi \rho)] y=x[(\lambda(s x) \rho) \psi] y=\left(x \psi^{\prime}\right) y
\end{aligned}
$$

which implies that $\left(\varphi^{\prime}, \psi^{\prime}\right) \in \Omega(S)$.

Further, for $x \in S$ and $\lambda x=(\lambda x) u(\lambda x)$, we have

$$
\begin{aligned}
\left(\lambda \varphi^{\prime}\right) x & =\lambda\left(\phi^{\prime} x\right)=\lambda\{[\varphi(\lambda(x u) \rho)] x\}=[(\lambda \varphi)(\lambda(x u) \rho)] x \\
& =[\varphi(\lambda(x u) \rho)] x=\phi^{\prime} x, \\
\left(\varphi^{\prime} \lambda\right) x & \left.=\varphi^{\prime}(\lambda x)=[\varphi(\lambda(x u) \rho))\right] x=\phi^{\prime} x
\end{aligned}
$$

which proves that $\phi^{\prime}=\lambda \varphi^{\prime}=\phi^{\prime} \lambda$; analogously $\psi^{\prime}=\rho \psi^{\prime}=\psi^{\prime} \rho$. Consequently $\left(\varphi^{\prime}, \psi^{\prime}\right) \in \omega \Omega(S) \omega$.

Finally let $x \in \omega S \omega, x=x u x$. Recall formula (3); then

$$
\begin{aligned}
\varphi^{\prime} x & =[\varphi(\lambda(x u) \rho)] x=\{\phi[(\lambda x)(u \rho)]\} x=\{\phi[(x \rho)(u \rho)]\} x \\
& =\{\phi[x(\lambda u \rho)]\} x=\varphi[x(\lambda u \rho) x]=\varphi[(x \rho)(u \rho) x] \\
& =\varphi[x u(\lambda x)]=\varphi(x u x)=\varphi x
\end{aligned}
$$

so that $\left.\phi^{\prime}\right|_{\omega S \omega}=\varphi$, analogously $\left.\psi^{\prime}\right|_{\omega S \omega}=\psi$. Therefore $\left(\varphi^{\prime}, \psi^{\prime}\right) \chi=(\varphi, \psi)$ and $\chi$ maps $\omega \Omega(S) \omega$ onto $\Omega(\omega S \omega)$.

CoRollary 1. Let $S$ be a regular semigroup. If $\omega \in E_{\Omega(S)}$, then $\omega \Omega(S) \omega \cap \Pi(S)$ is a densely embedded ideal of $\omega \Omega(S) \omega$.

Proof. Let $\pi: S \rightarrow \Omega(S)$ be the canonical homomorphism. It is easy to verify that

$$
\Pi(\omega S \omega) \cong \omega S \omega \cong \pi(\omega S \omega)=\omega \Omega(S) \omega \cap \Pi(S) .
$$

On the other hand, $\Pi(\omega S \omega)$ is a densely embedded ideal of $\Omega(\omega S \omega)$, which is in turn isomorphic to $\omega \Omega(S) \omega$ by the theorem.

CoRollary 2. If $\Omega(S)$ is a regular semigroup, and $\omega \in E_{\Omega(S)}$, then $\Omega(\omega S \omega)$ is a regular semigroup.

Proof. This follows from the theorem since $\Omega(\omega S \omega) \cong \omega \Omega(S) \omega$ and any maximal submonoid of a regular semigroup is regular.

LEMma 1. If $S$ is a regular semigroup and $\omega \in E_{\Omega(S)}$, then $\omega S \omega$ is a regular semigroup. 
Proof. Let $x \in \omega S \omega$ and $x^{\prime}$ be an inverse of $x$. Then

$$
\left.x=x x^{\prime} x=(x \rho) x^{\prime}(\lambda x)=x\left(\lambda x^{\prime}\right) \rho\right) x
$$

which shows that $\omega S \omega$ is regular.

COROLlaRY. If $S$ is an inverse semigroup (resp. a semilattice of groups) and $\omega \in E_{\Omega(S)}$, then both $\omega S \omega$ and $\Omega(\omega S \omega)$ are inverse semigroups (resp. semilattices of groups).

Proof. In view of the lemma, the assertion follows easily from ([5], V.4.6) (resp. V.6.6).

3. Rees matrix semigroups. The theorem of this section relates subdirect irreducibility of a maximal subgroup of a Rees matrix semigroup $S$ with that of a number of subsemigroups of $\Omega(S)$. We start with a general discussion and a string of lemmas.

Throughout this section we fix a (regular) Rees matrix semigroup $S=\mathscr{C}^{\circ}(I, G, M ; P)$. We outline briefly a construction of $\Omega(S)$, see ([5], V.3). For a partial transformation $\alpha$ on $I$, whose domain is denoted by $\boldsymbol{d} \alpha$, and a function $\varphi$ mapping $d \alpha$ into $G$, the mapping $\lambda$ defined by

$$
\lambda(i, g, \mu)=(\alpha i,(\varphi i) g, \mu) \quad \text { if } \quad i \in \boldsymbol{d} \alpha
$$

and $\lambda(i, g, \mu)=0$ otherwise, is a left translation of $S$; analogously

$$
(i, g, \mu) \rho=(i, g(\mu \psi), \mu \beta) \text { if } \mu \in \boldsymbol{d} \beta
$$

and $(i, g, \mu) \rho=0$ otherwise, is a right translation of $S$; they are linked if and only if

$$
\left\{\begin{aligned}
i \in d \alpha, p_{\mu(\alpha i)} \neq 0 & \Longleftrightarrow \mu \in d \beta, p_{(\mu \beta) i} \neq 0 \\
& \Longrightarrow p_{\mu(\alpha i)}(\phi i)=(\mu \psi) p_{(\mu \beta) i} .
\end{aligned}\right.
$$

In such a case, we write $\omega=(\lambda, \rho) \sim(\alpha, \phi ; \beta, \psi)$. Conversely, every bitranslation of $S$ is of this form for unique parameters $\alpha, \varphi, \beta, \psi$. It is easy to verify that $\omega^{2}=\omega$ if and only if

$$
\left.\alpha\right|_{r \alpha}=\iota_{r \alpha},\left.\varphi\right|_{r \alpha}: r \alpha \longrightarrow 1,\left.\beta\right|_{r \beta}=\iota_{r \beta},\left.\psi\right|_{r \beta}: r \beta \longrightarrow 1
$$

where $r \alpha$ is the range of $\alpha, \iota_{r \alpha}$ is the identity mapping on $r \alpha, 1$ is the identity of $G$, etc. With this notation, we have

Lemma 2. If $\omega \in E_{\Omega(S)}$, then $\omega S \omega=\mathscr{M}^{\circ}\left(\boldsymbol{r} \alpha, G, \boldsymbol{r} \beta ; P^{\omega}\right)$ where $P^{\omega}$ is the restriction of $P$ to $r \beta \times r \alpha$. 
Proof. Indeed, for $0 \neq(i, g, \mu) \in S$, we have

$$
\begin{aligned}
(i, g, \mu) \in \omega S \omega & \Longrightarrow(i, g, \mu)=\lambda(i, g, \mu)=(i, g, \mu) \rho \\
& \Longrightarrow(i, g, \mu)=(\alpha i,(\varphi i) g, \mu)=(i, g(\mu \psi), \mu \beta) \\
& \Longrightarrow i=\alpha i, \phi i=1, \mu \psi=\mu, \mu \beta=\mu \\
& \Longrightarrow i \in \boldsymbol{r} \alpha, \mu \in \boldsymbol{r} \beta .
\end{aligned}
$$

By Lemma 1, $\omega S \omega$ is regular, hence the sandwich matrix $P^{\omega}$ has a nonzero element in each row and each column.

If the sandwich matrix $P$ has no two distinct rows (or columns) which have the corresponding entries simultaneously nonzero, then $P$ (and also $S$ ) is said to have no contractions, see $([3], \S 6)$. The importance of this notion stems from the fact that these are precisely completely 0 -simple semigroups all of whose proper congruences are contained in $\mathscr{H}$.

Lemma 3. Let the notation be as in Lemma 2. If $P$ has no contractions, then neither does $P^{\omega}$.

Proof. Let $i, j \in \boldsymbol{r} \alpha$ and assume that

$$
p_{\mu i} \neq 0 \Longleftarrow p_{\mu j} \neq 0 \quad(\mu \in \boldsymbol{d} \beta) .
$$

Let $\mu \in M$ be such that $p_{\mu_{i}} \neq 0$. Now $i \in \boldsymbol{r} \alpha$ implies that $i \in d \alpha$ and $\alpha i=i$ since $\alpha^{2}=\alpha$. Hence $i \in \boldsymbol{d} \alpha$ and $p_{\mu(\alpha i)} \neq 0$ which by (4) implies that $\mu \in \boldsymbol{d} \beta$ and $p_{(\mu \beta)_{i}} \neq 0$. Here $\mu \beta \in \boldsymbol{r} \beta$ and $p_{(\mu \beta)_{i}} \neq 0$ so that by (5), we have $p_{(\mu \beta) j} \neq 0$. But then $\mu \in \boldsymbol{d} \beta$ and $p_{(\mu \beta) j} \neq 0$ and hence $j \in d \alpha$ and $p_{\mu(\alpha j)} \neq 0$ by (4). Since $\alpha j=j$, it follows that $p_{\mu j} \neq 0$. By symmetry, we conclude that

$$
p_{\mu i} \neq 0 \Longleftrightarrow p_{\mu j} \neq 0 \quad(\mu \in M),
$$

which by hypothesis that $P$ has no contractions implies that $i=j$. One proves symmetrically that for $\mu, \nu \in \boldsymbol{r} \beta$,

$$
p_{\mu_{i}} \neq 0 \Longleftarrow p_{\nu i} \neq 0 \quad(i \in r \alpha)
$$

implies $\mu=\nu$. Therefore $P^{\omega}$ has no contractions.

The next result is of general interest for extensions of regular semigroups.

Lemma 4. Let $V$ be an extension of a regular semigroup $S$. Then every congruence on $S$ contained in $\mathscr{H}$ can be extended to a congruence on $V$. 
Proof. Let $\sigma$ be a congruence on $S$ contained in $\mathscr{H}$ and $\tau$ be the equivalence relation on $V$ whose classes are the $\sigma$-classes and singletons $\{v\}$ with $v \in V \backslash S$. Then $\tau$ is a congruence if and only if for any $v \in V, a, b \in S, a \sigma b$ implies $v a \sigma v b$ and $a v \sigma b v$. Let $a, b \in S$ be such that $a \sigma b$. The hypothesis implies that $a \mathscr{H} b$, and thus $a=b x$ for some $x \in S$. Let $b^{\prime}$ be an inverse of $b$. Then

$$
a=b x=\left(b b^{\prime} b\right) x=b b^{\prime}(b x)=b b^{\prime} a,
$$

and thus for any $v \in V$, we have

$$
v a=v\left(b b^{\prime} a\right)=\left(v b b^{\prime}\right) a \sigma\left(v b b^{\prime}\right) b=v b
$$

since $v b b^{\prime} \in S$. A symmetric argument can be used to show that avobv. Consequently $\tau$ is a congruence and is obviously an extension of $\sigma$.

LEMma 5. Let $V$ be a dense extension of a semigroup S. If $S$ is subdirectly irreducible, then so is $V$. The converse holds if every congruence on $S$ can be extended to a congruence on $V$.

Proof. This is a part of ([5], III.5.19 Exerc. 5).

We can now prove the desired result.

TheOREM 2. Let $S=\mathscr{C l}^{\circ}(I, G, M ; P)$ and assume that $P$ has no contractions. Let $\omega \in E_{\Omega(S)}$ and $V$ be a subsemigroup of $\Omega(S)$ such that

$$
\omega \Omega(S) \omega \cap \Pi(S) \subseteq V \leqq \omega \Omega(S) \omega .
$$

Then $G$ and $V$ are simultaneously subdirectly reducible or irreducible.

Proof. We have mentioned above that the hypothesis that $P$ has no contractions is equivalent to $S$ having all proper congruences contained in $\mathscr{H}$ ([3], Proposition 6.2). Any one of the numerous descriptions of congruences on a Rees matrix semigroup can be used to easily show that the lattice of all congruences on $S$ contained in $\mathscr{H}$ is isomorphic to the lattice of all congruences (and thus normal subgroups) on $G$. Under our hypothesis this means that $G$ is subdirectly irreducible if and only if $S$ is.

By Lemma 3, the matrix $P^{\omega}$ has no contractions. The above argument for $S$ is now valid for $\omega S \omega$ in view of Lemma 2. Hence $G$ and $\omega S \omega$ are simultaneously subdirectly irreducible or not. By Lemma $1, \omega S \omega$ is regular. It follows that 
as in the proof of Corollary 1 to Theorem 1. According to the last reference, we also have that $\omega \Omega(S) \omega \cap \Pi(S)$ is a densely embedded ideal of $\omega \Omega(S) \omega$. Hence by ([5], III.5.6), $V$ given in the statement of the theorem is a dense extension of $\omega \Omega(S) \omega \cap \Pi(S)$. Since the last semigroup has no contractions, its proper congruences are contained in $\mathscr{H}$, so by Lemma 4, are extendible to $V$. But then Lemma 5 asserts that $\omega \Omega(S) \omega \cap \Pi(S)$ is subdirectly irreducible if and only if $V$ is.

Now a combination of the statements concerning $G$ and $\omega S \omega$, (6), and $\omega \Omega(S) \omega \cap \Pi(S)$ and $V$, establishes the theorem.

Note that for $\omega=\left(c_{S}, \iota_{S}\right)$, the identity bitranslation, we may take $V=\Pi(S)$ (and $\Pi(S) \cong S$ ), or $V=\Omega(S)$. Also for any nonzero idempotent $e$ of $S$, the bitranslation $\omega=\left(\lambda_{e}, \rho_{e}\right)$ gives for $\omega S \omega$ the maximal subgroup $G_{e}$ of $S$ with identity $e$ (and $G_{e} \cong G$ ). Also observe that we have used Theorem 1 via its Corollary 1.

4. Retracts. A subsemigroup $T$ of a semigroup $S$ is a retract (of $S$ ) if there exists a homomorphism $\varphi$ of $S$ onto $T$ which leaves all elements of $T$ fixed; $\varphi$ is then a retraction. We discuss here regular semigroups in which all its maximal submonoids are retracts. A related condition will be expressed by means of bitranslations; for this reason we introduce

Definition. Let $S$ be a semigroup and $(\lambda, \rho) \in E_{\Omega(S)}$ such that $(\lambda x) \rho=\lambda(x \rho)$ for all $x \in S$ (so we can write $\lambda x \rho$ without ambiguity). The mapping

$$
[\lambda, \rho]: x \longrightarrow \lambda x \rho \quad(x \in S)
$$

is said to be induced by $(\lambda, \rho)$.

Lemma 6. Consider the following conditions on a semigroup S.

(a) For any $a, b \in S, e \in E_{S}$, eabe = eaebe.

(b) Every maximal submonoid of $S$ is a retract.

(c) Every idempotent inner bitranslation on $S$ induces a retraction.

Then (a) and (b) are equivalent; (c) implies (a); and (a) implies (c) if $S$ is weakly reductive.

Proof. Straightforward.

Recall that an idempotent semigroup satisfying the condition 
(a) in Lemma 6 is called a regular band. We are now ready for the theorem of this section.

THEOREM 3. Let $S$ be a regular semigroup. If $S$ satisfies condition (a) in Lemma 6, then it also satisfies the following conditions.

(d) $S$ is completely regular.

(e) Every idempotent bitranslation induces a retraction.

(f) Idempotents of $S$ form a regular band.

Proof. (d). Let $a^{\prime}$ be an inverse of an element $a$ of $S$. Then

$$
a=\left(\alpha \alpha^{\prime}\right) \alpha \alpha^{\prime}\left(\alpha \alpha^{\prime}\right) \alpha=\left(\alpha \alpha^{\prime}\right) \alpha\left(\alpha \alpha^{\prime}\right) \alpha^{\prime}\left(\alpha \alpha^{\prime}\right) a \in \alpha^{2} S \alpha
$$

which by ([5], IV.1.6) implies that $S$ is completely regular.

(e) Let $(\lambda, \rho) \in E_{\Omega(S)}, x, y \in S$. Using part (d), for any element $z \in S$, we let $z^{\prime}$ be the inverse of $z$ in the maximal subgroup of $S$ containing $z$. We compute

$$
\begin{aligned}
\lambda(x y) \rho & =[\lambda(x y) \rho][\lambda(x y) \rho]^{\prime}[\lambda(x y) \rho] \\
& =\left\{\left[(\lambda x)(\lambda x)^{\prime}\right](\lambda x)(y \rho)[\lambda(x y) \rho]^{\prime}\left[(\lambda x)(\lambda x)^{\prime}\right]\right\}(\lambda x)(y \rho) \\
& =\left\{\left[(\lambda x)(\lambda x)^{\prime}\right](\lambda x)\left[(\lambda x)(\lambda x)^{\prime}\right](y \rho)[\lambda(x y) \rho]^{\prime}\left[(\lambda x)(\lambda x)^{\prime}\right]\right\}(\lambda x)(y \rho) \\
(7) & =\left\{\left[(\lambda x)(\lambda x)^{\prime}\right](\lambda x \rho)\left[(\lambda x)(\lambda x)^{\prime}\right](y \rho)[\lambda(x y) \rho]^{\prime}\left[(\lambda x)(\lambda x)^{\prime}\right]\right\}(\lambda x)(y \rho) \\
& =\left\{\left[(\lambda x)(\lambda x)^{\prime}\right](\lambda x \rho)(y \rho)[\lambda(x y) \rho]^{\prime}\left[(\lambda x)(\lambda x)^{\prime}\right]\right\}(\lambda x)(y \rho) \\
& =(\lambda x)(\lambda y \rho)[\lambda(x y) \rho]^{\prime}[\lambda(x y) \rho] \\
& =(\lambda x \rho)(\lambda y \rho)[\lambda(x y) \rho]^{\prime}[\lambda(x y) \rho]
\end{aligned}
$$

analogously

$$
\lambda(x y) \rho=[\lambda(x y) \rho][\lambda(x y) \rho]^{\prime}(\lambda x \rho)(\lambda y \rho) .
$$

On the other hand,

$$
\begin{aligned}
(\lambda x \rho)(\lambda y \rho)= & (\lambda x \rho)(\lambda y \rho)[(\lambda x \rho)(\lambda y \rho)]^{\prime}(\lambda x \rho)(\lambda y \rho) \\
= & {\left[(\lambda x \rho)(\lambda x \rho)^{\prime}\right](\lambda x \rho)(y \rho)[(\lambda x \rho)(\lambda y \rho)]^{\prime}\left[(\lambda x \rho)(\lambda x \rho)^{\prime}\right](\lambda x \rho)(\lambda y \rho) } \\
= & {\left[(\lambda x \rho)(\lambda x \rho)^{\prime}\right](\lambda x \rho)\left[(\lambda x \rho)(\lambda x \rho)^{\prime}\right](y \rho) } \\
& \times[(\lambda x \rho)(\lambda y \rho)]^{\prime}\left[(\lambda x \rho)(\lambda x \rho)^{\prime}\right](\lambda x \rho)(\lambda y \rho) \\
= & {\left[(\lambda x \rho)(\lambda x \rho)^{\prime}\right](\lambda x)\left[(\lambda x \rho)(\lambda x \rho)^{\prime}\right](y \rho) } \\
& \times[(\lambda x \rho)(\lambda y \rho)]^{\prime}\left[(\lambda x \rho)(\lambda x \rho)^{\prime}\right](\lambda x \rho)(\lambda x \rho) \\
= & (\lambda x \rho)(\lambda x \rho)^{\prime}[\lambda(x y) \rho][(\lambda x \rho)(\lambda y \rho)]^{\prime}(\lambda x \rho)(\lambda y \rho) .
\end{aligned}
$$

The conjunction of (7) and (9) shows that $\lambda(x y) \rho$ and $(\lambda x \rho)(\lambda y \rho)$ are $\mathscr{J}$-related. Since $S$ is completely regular, they are contained in a completely simple subsemigroup of $S$. Hence (7) and (8) imply that 
they are also contained in the same maximal subgroup $G$ of $S$. But then $[\lambda(x y) \rho]^{\prime}[\lambda(x y) \rho]$ must the identity of $G$, which together with (7) shows that $\lambda(x y) \rho=(\lambda x \rho)(\lambda y \rho)$. This is evidently equivalent to the statement that the bitranslation $(\lambda, \rho)$ induces a retraction.

(f) It suffices to show that idempotents of $S$ form a subsemigroup. Using a Rees matrix representation of a completely simple semigroup $T$, it is an easy exercise to show that condition (a) in Lemma 6 implies that $E_{T}$ is a subsemigroup of $T$. Since $S$ is a semilattice of completely simple semigroups, ([5], IV.3.7) implies that $E_{S}$ is a subsemigroup of $S$.

Comparing Lemma 6 with Theorem 3, we see that if in a regular semigroup every idempotent inner bitranslation induces a retraction, then so does every idempotent bitranslation. The semigroup $S$ of all transformations on a set of two elements is regular and trivially satisfies condition (a); in this semigroup $\mathscr{H}$ is not a congruence. However, if $S$ is a regular semigroup satisfying (a) in which $\mathscr{H}$ is a congruence, then it follows easily from ([4], Theorem 3.2) that $S$ is a subdirect (even spined) product of a semilattice of groups and a regular band. Conversely, it is easy to see that a regular semigroup $S$ which is a subdirect product of a semilattice of groups and a regular band must satisfy (a) and $\mathscr{H}$ is a congruence on $S$. It seems unlikely that conditions (d) and (f) in Theorem 3 imply condition (a).

One might conjecture that if a regular semigroup $S$ satisfies condition (a) and $\Omega(S)$ is regular, then $\Omega(S)$ also satisfies (a). This, however, is far from being the case. If $T$ is the semigroup of all transformations on a set with at least three elements, then the constants in $T$ form an ideal $S$ of $T$ such that: $(\alpha) S$ is a left (if the transformations are written on the left) zero semigroup, thus regular and satisfying (a), $(\beta) \Omega(S) \cong T$ so that $\Omega(S)$ is a regular semigroup. If $\Omega(S)$ satisfied (a), then by Theorem 3 , it would have to be completely regular. But $T$ is not completely regular, so $\Omega(S)$ does not satisfy (a).

5. Examples. The following examples illustrate some of the applications of Theorems 1 and 2. The proofs of many assertions that follow are either omitted or can be found in [5].

(a) The semigroup $\mathscr{T}(X)$ of transformations on a set $X$ (written on the left). For the constants $\mathscr{T}_{0}(X)$, we have

$$
\mathscr{T}_{0}(X) \cong \mathscr{L C}(X, 1,\{X\} ; P)
$$

with $P=\left(p_{X a}\right), \quad p_{X a}=1$ (right zero semigroup on $X$ ), 1 is a one element group, 


$$
\mathscr{T}(X) \cong \Omega(\mathscr{M}(X, 1,\{X\}, P))
$$

For any $\alpha \in E_{\mathscr{T}(X)}$, we have

$$
\alpha \mathscr{T}(X) \alpha \cong \Omega\left(\mathscr{L}\left(r \alpha, 1,\{r \alpha\} ; P^{\alpha}\right)\right) \cong \mathscr{T}(\alpha X),
$$

where $P^{\alpha}$ is essentially the restriction of $P$.

(b) The semigroup $\mathscr{F}(X)$ of partial transformations on a set $X$ (written on the left). For the (partial) constants $\mathscr{F}_{0}(X)$, we have

$$
\mathscr{F}_{0}(X) \cong \mathscr{M}^{0}\left(X, 1, \mathfrak{P}(X) ; P_{X}\right)
$$

where $\mathfrak{P}(X)$ is the set of all nonempty subsets of $X, P_{X}=\left(p_{A a}\right)$, $p_{A a}=1$ if $a \in A, p_{A a}=0$ if $a \notin A$;

$$
\mathscr{F}(X) \cong \Omega\left(\mathscr{C}^{0}\left(X, 1, \mathfrak{P}(X) ; P_{X}\right)\right) \text {. }
$$

For any $\alpha \in E_{\mathscr{F}(X)}$, we have

$$
\alpha \mathscr{F}(X) \alpha \cong \Omega\left(\mathscr{C}^{0}\left(r \alpha, 1, r \beta ; P^{\alpha}\right)\right)
$$

where $\beta$ is a partial transformation on $\mathfrak{P}(X)$ with

$$
\begin{aligned}
& \boldsymbol{d} \beta=\{B \subseteq X \mid B \cap \boldsymbol{r} \alpha \neq \varnothing\}, \\
& B \beta=\{x \in \boldsymbol{d} \alpha \mid \alpha x \in B\} \quad \text { if } B \in \boldsymbol{d} \beta, \\
& \boldsymbol{r} \beta=\{B \mid B \cap \boldsymbol{r} \alpha \neq \varnothing\},
\end{aligned}
$$

and $P^{\alpha}$ is essentially the restriction of $P_{X}$. It can be proved that

$$
\mathscr{M}^{0}\left(\boldsymbol{r} \alpha, 1, r \beta ; P^{\alpha}\right) \cong \mathscr{M}^{\circ}\left(\boldsymbol{r} \alpha, 1, \mathfrak{\beta}(\boldsymbol{r} \alpha) ; P_{\mathrm{r} \alpha}\right)
$$

and thus (10)-(12) yield

$$
\alpha \mathscr{F}(X) \alpha \cong \mathscr{F}(r \alpha) .
$$

It can be shown that none of the Rees matrix semigroups here has contractions. Hence all these semigroups are subdirectly irreducible.

(c) The semigroup $\mathscr{S}(V)$ of linear transformations on a (left) vector space $V$ (written on the right). We will use the notation and results of ([6], I.2). The semigroup $\mathscr{S}_{0}(V)$ of linear transformations of rank $\leqq 1$ has the property

$$
\mathscr{S}_{0}(V) \cong \mathscr{M}^{0}\left(I_{V^{*}}, \mathscr{M S}^{-}, I_{V} ; P\right)
$$

and

$$
\mathscr{S}(V) \cong \Omega\left(\mathscr{L}^{0}\left(I_{V^{*}}, \mathscr{M S}^{-}, I_{V} ; P\right)\right) .
$$

For any $0 \neq \alpha \in E_{G(V)}$, we have

$$
\alpha \mathscr{S}(V) \alpha \cong \Omega\left(\mathscr{C}^{0}\left(I_{\alpha^{*} V^{*}}, \mathscr{C A}^{-}, I_{V \alpha} ; P^{\alpha}\right)\right) \cong \mathscr{S}(V \alpha) \text {. }
$$


It can be shown that the matrix $P$ has no contractions. Consequently $\mathscr{C A}^{-}$(the multiplicative group of nonzero elements of the division ring $\Delta$ of the vector space $V), \mathscr{S}_{0}(V)$ and $\mathscr{S}(V)$ are simultaneously subdirectly reducible or irreducible.

(d) Brandt semigroups $S=\mathscr{C}^{0}(X, G, X ; \Delta)$. For $0 \neq \omega \in E_{\Omega(S)}$, we have

$$
\omega \Omega(S) \omega \cong \Omega\left(\mathscr{C}^{\circ}(r \alpha, G, r \alpha ; \Delta)\right) .
$$

Let $\mathscr{I}(X)$ be the semigroup of partial 1-1 transformations on $X$, and $\mathscr{J}_{0}(X)$ be the partial 1-1 constants on $X$. Then

$$
\begin{aligned}
& \mathscr{F}_{0}(X) \cong \mathscr{L}^{\circ}(X, 1, X ; \Delta) \\
& \mathscr{F}(X) \cong \Omega\left(\mathscr{L}^{\circ}(X, 1, X ; \Delta)\right),
\end{aligned}
$$

and if $0 \neq \alpha \in E_{\mathcal{J}(X)}$, then

$$
\alpha \mathscr{F}(X) \alpha \cong \Omega\left(\mathscr{C}^{\circ}(r \alpha, 1, r \alpha ; \Delta)\right) \cong \mathscr{I}(r \alpha) .
$$

None of these Rees matrix semigroups has contractions; hence $G$, $\mathscr{C}^{\circ}(X, G, X ; \Delta), \Omega\left(\mathscr{C}^{\circ}(X, G, X ; \Delta)\right)$ are simultaneously subdirectly reducible or irreducible. In particular both $\mathscr{I}_{0}(X)$ and $\mathscr{I}(X)$ are subdirectly irreducible.

(e) The semigroup $\mathscr{B}(X)$ of binary relations on a set $X$. For the semigroup $\mathscr{R}(X)$ of all rectangular binary relations on $X$, we have

$$
\mathscr{R}(X) \cong \mathscr{L}^{\circ}(\mathfrak{P}(X), 1, \mathfrak{P}(X) ; P)
$$

with $p_{A B}=1$ if $A \cap B \neq \varnothing$ and $p_{A B}=0$ otherwise. Further,

$$
\mathscr{B}(X) \cong \Omega\left(\mathscr{C}^{0}(\mathfrak{P}(X), 1, \mathfrak{P}(X) ; P)\right) \text {. }
$$

Let $0 \neq \sigma \in E_{\mathscr{B}(X)}$. Then

$$
\sigma \mathscr{B}(X) \sigma \cong \Omega\left(\mathscr{C}^{0}\left(\boldsymbol{r} \alpha, 1, \boldsymbol{r} \beta ; P^{\sigma}\right)\right)
$$

where $\alpha$ and $\beta$ are partial transformations on $X$ for which

$$
\begin{aligned}
& d \alpha=\{A \subseteq X \mid(X \times A) \cap \sigma \neq \varnothing\}, \\
& \alpha A=\{x \in X \mid x \sigma y \text { for some } y \in A\} \text { if } \quad A \in d \alpha,
\end{aligned}
$$

and $\boldsymbol{d} \beta$ and $B \beta$ are defined symmetrically, $P^{\alpha}$ is essentially the restriction of $P$; see [1]. We may let $Y=(r \beta \cup\{\varnothing\}) \backslash(X \sigma)$ and

$$
T=\mathscr{C}^{0}(Y, 1, r \beta ; Q)
$$

with $Q=\left(q_{A B}\right), q_{A B}=1$ if $A \nsubseteq B$ and $q_{A B}=0$ otherwise. Using some results of Zareckii [7], one can show that

$$
\mathscr{C}^{0}\left(\boldsymbol{r} \alpha, 1, r \beta ; P^{o}\right) \cong \mathscr{C}^{0}(Y, 1, \boldsymbol{r} \beta ; Q)
$$


so that

$$
\sigma \mathscr{B}(X) \sigma \cong \Omega\left(\mathscr{C}^{\circ}(Y, 1, \boldsymbol{r} \beta ; Q)\right)
$$

None of these Rees matrix semigroups has contractions. Hence all these semigroups are subdirectly irreducible. In particular, this implies ([7], Proposition 4.4). Also Corollary 1 to Theorem 1 for $S=\mathscr{R}(X)$ implies ([7], Theorem 3.2). The semigroup $T$ in (13) is particularly interesting since it can be constructed directly by means of a completely distributive lattice, which then yields an abstract characterization of maximal submonoids of $\mathscr{B}(X)$, see [7].

\section{REFERENCES}

1. M. Petrich, The translational hull and semigroups of binary relations, Glasgow Math. J., 9 (1968), 12-21.

2. - The translational hull in semigroups and rings, Semigroup Forum, 1 (1970), 283-360.

3. - Regular semigroups satisfying certain conditions on idempotents and ideals, Trans. Amer. Math. Soc., 179 (1972), 245-269.

4. - Regular semigroups which are subdirect products of a band and a semilattice of groups, Glasgow Math. J., 14 (1973), 27-49.

5. - Introduction to Semigroups, Merrill, Columbus, 1973.

6. - Rings and Semigroups, Lecture Notes in Math. No. 380, Springer, New York, Heidelberg, Berlin, 1974.

7. K. A. Zareckiil, Maximal submonoids of the semigroup of binary relations, Semigroup Forum, 9 (1974), 196-208.

Received December 19, 1974.

Pennsylvania State University

UNIVERSITY PARK, PA 16802 



\section{PACIFIC JOURNAL OF MATHEMATICS}

\section{EDITORS}

RICHARD ARENS (Managing Editor)

University of California

Los Angeles, California 90024

C. W. CURTIS

University of Oregon

Eugene, OR 97403

C. C. MOORE

University of California

Berkeley, CA 94720

\section{J. DugundJI}

Department of Mathematics University of Southern Californıa Los Angeles, California 90007

R. FinN AND J. Milgram Stanford University Stanford, California 94305

\section{ASSOCIATE EDITORS}

E. F. BECKENBACH

B. H. NEUMANN

F. WOLF

K. YOSHIDA

\section{SUPPORTING INSTITUTIONS}

UNIVERSITY OF BRITISH COLUMBIA CALIFORNIA INSTITUTE OF TECHNOLOGY UNIVERSITY OF CALIFORNIA MONTANA STATE UNIVERSITY UNIVERSITY OF NEVADA, RENO NEW MEXICO STATE UNIVERSITY OREGON STATE UNIVERSITY UNIVERSITY OF OREGON OSAKA UNIVERSITY
UNIVERSITY OF SOUTHERN CALIFORNIA STANFORD UNIVERSITY UNIVERSITY OF TOKYO UNIVERSITY OF UTAH WASHINGTON STATE UNIVERSITY UNIVERSITY OF WASHINGTON AMERICAN MATHEMATICAL SOCIETY NAVAL WEAPONS CENTER 


\section{Pacific Journal of Mathematics}

\section{Vol. 71, No. $1 \quad$ November, 1977}

Charalambos D. Aliprantis and Owen Sidney Burkinshaw, On universally complete Riesz spaces ............................. 1

Stephen Richard Bernfeld and Jagdish Chandra, Minimal and maximal solutions of nonlinear boundary value problems .................

John H. E. Cohn, The length of the period of the simple continued fraction of

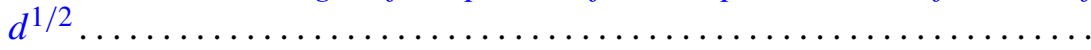

Earl Vern Dudley, Sidon sets associated with a closed subset of a compact abelian group .................................... 33

Larry Finkelstein, Finite groups with a standard component of type $J_{4} \ldots \ldots$

Louise Hay, Alfred Berry Manaster and Joseph Goeffrey Rosenstein, Concerning partial recursive similarity transformations of linearly ordered sets .......................................

Richard Michael Kane, On loop spaces without $p$ torsion. II ............

William A. Kirk and Rainald Schoneberg, Some results on

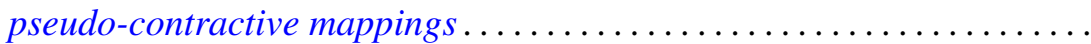

Philip A. Leonard and Kenneth S. Williams, The quadratic and quartic character of certain quadratic units. I. .

Lawrence Carlton Moore, A comparison of the relative uniform topology and the norm topology in a normed Riesz space .................

Mario Petrich, Maximal submonoids of the translational hull 119

Mark Bernard Ramras, Constructing new R-sequences . . .

Dave Riffelmacher, Multiplication alteration and related rigidity properties

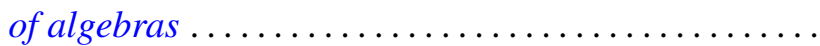

Jan Rosiński and Wojbor Woyczynski, Weakly orthogonally additive functionals, white noise integrals and linear Gaussian stochastic processes.

Ryōtarō Satō, Invariant measures for ergodic semigroups of operators

Peter John Slater and William Yslas Vélez, Permutations of the positive integers with restrictions on the sequence of differences...

Edith Twining Stevenson, Integral representations of algebraic cohomology classes on hypersurfaces ........................

Laif Swanson, Generators of factors of Bernoulli shifts . 